\title{
ON THE INCLUSION OF A BOUNDED CONVERGENCE FIELD IN THE SPACE OF ALMOST CONVERGENT SEQUENCES
}

\author{
by ROBERT E. ATALLA
}

(Received 24 December, 1969; revised 1 September, 1970)

Introduction. Let $T=\left(t_{m n}\right)$ be a regular matrix, and $C_{T}$ be its bounded convergence field. Necessary and sufficient conditions for $C_{T}$ to contain the space of almost convergent sequences are well known. (See, e.g., [7, p. 62]). G. M. Petersen has suggested as a problem for research the discovery of necessary and sufficient conditions for the reverse inclusion: When is $C_{T}$ contained in the space of almost convergent sequences? [7, p. 137, research problem 9]. In this paper we deal with this question in a more general context. First we need some notation.

Letting $T$ be as above, let $C^{*}(N)$ be the bounded real valued functions on the positive integers. We define $m_{T}$ as the set of " $T$-invariant means", i.e. the set of positive linear functionals $\phi$ on $C^{*}(N)$ such that $\phi(e)=1$ (where $e$ is the unit function), $\phi \circ T=\phi$, and $\phi(f)=0$ whenever $\lim _{n \rightarrow \infty} f(n)=0$. We define $V_{T}=\left\{f \in C^{*}(N): \phi_{1}(f)=\phi_{2}(f)\right.$ for all $\phi_{1}$, $\left.\phi_{2} \in m_{T}\right\}$, and write $m_{T}(f)$ for this common value. It follows easily from our assumptions on $m_{T}$ that $C_{T} \subset V_{T}$ consistently, in the sense that $m_{T}(f)=T-\lim (f)$ for each $f \in C_{T}$. Notice that $V_{T}$ is the usual space of almost convergent functions when $T$ is the shift matrix transformation $T f(n)=f(n+1)$.

Now let $T=\left(t_{m n}\right)$ and $S=\left(s_{m n}\right)$ be non-negative regular matrices, and consider the following possible relations.

(I) $V_{T} \subset C_{S}$ consistently,

(II) $V_{T} \subset V_{S}$ consistently,

(III) $C_{T} \subset V_{S}$ consistently.

Since $C_{T} \subset V_{\mathrm{T}}$ and $C_{\mathrm{S}} \subset V_{S}$, these three relations are in decreasing order of strength, i.e. (I) implies (II), and (II) implies (III). If $S$ is the shift matrix, then(III) is related to Petersen's problem.

In Section 2, we give necessary and sufficient conditions for (I) and (II). Actually this has already been done for a certain class of permutation matrices by Raimi [8] and Dean and Raimi [4], and the proofs in the general case are largely adaptations of their proofs. An interesting side result is obtained ( 2.3 below), namely that, for sequences of linear operators induced on $C(\beta N \mid N)$ by regular matrix operators on $C^{*}(N)$, convergence in norm is equivalent to convergence in the strong operator topology [3, p. 34].

In Section 3 we give a condition sufficient for (III). In Section 4 we show that, if $T$ is suitably restricted, then this condition is also necessary.

1. Preliminaries. In this section we introduce some notations and lemmas needed in the sequel.

1.1. Notation. If $f \in C^{*}(N)$, let $f^{\prime}$ be its extension to $\beta N$, and $f^{*}=f^{\prime} \mid \beta N \backslash N$. If $A \subset N$, let $A^{\prime}$ be its closure in $\beta N$ and $A^{*}=A^{\prime} \cap \beta N \backslash N$. Then $N^{*}=\beta N \backslash N$, and $C\left(N^{*}\right)$ is the space of continuous real functions on $N^{*}$. 
If $T=\left(t_{m n}\right)$ is non-negative regular and $C_{0}$ the elements of $C^{*}(N)$ with limit zero, then $T\left(C_{0}\right) \subset C_{0}$. Hence $T$ induces a positive linear operator $T_{1}$ on $C\left(N^{*}\right)$ defined by $T_{1}\left(f^{*}\right)=(T f)^{*}$. The norm of this operator on $C^{*}(N)$ is given by $\left\|T_{1}\right\|=\limsup _{m \rightarrow \infty} \sum_{k} t_{m k}=1$ (cf. [1] or [2]). The regularity of $T$ means that $T_{1} e=e$, where $e$ is the unit in $C\left(N^{*}\right)$.

$T=\left(t_{m n}\right)$ and $S=\left(s_{m n}\right)$ are called equivalent if $\lim _{m \rightarrow \infty} \sum_{k}\left|t_{m k}-s_{m k}\right|=0$.

If $T$ is regular, then $T$ is equivalent to a truncated matrix $S$, i.e. one such that there exists $m(1)<m(2)<\ldots$ and $n(1)<n(2)<\ldots$ such that, if $m \in[m(k), m(k+1))$, then $s_{m n}=0$ for $n \notin[n(k), n(k+2))[7$, p. 82]. There is no loss in generality in assuming that our matrices have truncated form, for if $S$ and $T$ are equivalent, then for each $f \in C^{*}(N), \lim _{m \rightarrow \infty}(T f-S f)(m)=0$, and hence $T_{1}=S_{1}$ on $C\left(N^{*}\right), C_{T}=C_{S}, V_{T}=V_{S}$, and $m_{T}=m_{S}$. Note that the sum and product of truncated matrices are truncated. Note also that $(S \circ T)_{1}=S_{1} \circ T_{1}$ for $S$ and $T$ regular.

If $n \in N, \varepsilon_{n}$ is the functional on $C^{*}(N)$ defined by $\varepsilon_{n}(f)=f(n)$; and if $w \in N^{*}, \varepsilon_{w}$ is defined on $C\left(N^{*}\right)$ by $\varepsilon_{w}(f)=f(w)$. Then $\varepsilon_{n} \circ T(f)=T f(n)$, etc. is $T_{n}$.

If $T$ is a matrix, let $T_{n}=(1 / n) \sum_{k=1}^{n} T^{k}$, where $T^{k}$ is the $k$ th iterate of $T$. If $T$ is regular, so Finally we define

$$
\begin{aligned}
Z_{T} & =\left\{f \in C^{*}(N): m_{T}(f)=0\right\}, \\
K(T) & =\left\{f \in C_{T}: T-\lim (f)=0\right\} \\
& =\left\{f \in C_{T}: T_{1} f^{*}=0\right\} .
\end{aligned}
$$

The following lemmas are mainly slight modifications of those which occur in [4] and [8]. The main difference is that we assume an extra property for our $T$-invariant means $m_{T}$, namely that $m_{T}(f)=0$ for all $f \in C_{0}$, and hence $\phi(f)=\phi(g)$ for all $\phi \in m_{T}$, whenever $f-g \in C_{0}$. This leads to our substituting the norm of $f^{*}$ on $N^{*}$ for that of $f$ on $N$, and that of $T_{1}$ on $C\left(N^{*}\right)$ for that of $T$ on $C^{*}(N)$.

1.2. Lemma. $m_{T}$ is the weak-* closed convex hull (in the dual $C^{*}(N)^{\prime}$ of $C^{*}(N)$ ) of the collection of all functionals of the form

$$
\phi=\lim \left\{\varepsilon_{p(a)} \circ T_{n(a)}: a \in A\right\},
$$

where $A$ is a directed set, and

$$
\lim \{p(a): a \in A\}=\lim \{n(a): a \in A\}=\infty .
$$

Proof. On page 471 of [4] it is noted that $\left\{T_{n}: n \in N\right\}$ is a " net of averages converging to $T$-invariance ", where the natural order is taken for $N$. Hence the result is just a restatement of Theorem 2.1 of [4], except for the assumption that $\lim \{p(a): a \in A\}=\infty$. But this is an easy consequence of our assumption that $m_{T}(f)=0$ for all $f \in C_{0}$. (Using this fact it is easy to check that in the first and second lemmas in the proof of Theorem 2.1 of [4], the expression " $\lim \sup \left(p^{\prime}, S_{\alpha} f\right)$ " may be replaced by " $\lim \lim \sup \left(p^{\prime}, S_{\alpha} f\right)$ ”.) 
1.3. LemMA. (a) $C_{T} \subset V_{T}$,

(b) $V_{T}=Z_{T} \oplus\{k e: k$ scalar $\}$,

(c) $C_{T}=K(T) \oplus\{k e: k$ scalar $\}$.

Proof. (a) if $\lim _{n \rightarrow \infty}(T f)(n)=k$, then by regularity of $T, \lim _{n \rightarrow \infty}(T(f-k e))(n)=0$, whence for each $\phi \in m_{T}, \phi(f-k e)=\phi \circ T(f-k e)$, or $\phi(f)=\phi(k e)=k$. (b) This follows since $\phi(e)=1$ for all $\phi \in m_{T}$. (c) This follows by regularity of $T$.

1.4. LEMMA (cf. Theorem 3.2 of [4]). If $f \in C^{*}(N)$, the following are equivalent:

(a) $f \in Z_{r}$,

(b) $\lim _{n \rightarrow \infty}\left(T_{n}\right)_{1} f^{*}=0$, where $\left(T_{n}\right)_{1}$ is the operator on $C\left(N^{*}\right)$ induced by $T_{n}$,

(c) $f^{*}$ belongs to the norm closed linear hull of $\left\{T_{1} g^{*}-g^{*}: g \in C^{*}(N)\right\}$.

Proof. (a) implies (b). If (b) fails, then there exists $\varepsilon>0$ and $n(1)<n(2)<\ldots$ such that $\left(T_{n(k)}\right)_{1} f^{*}>\varepsilon$.

Hence there exist $p(1)<p(2)<\ldots$ such that $\left|\varepsilon_{p(k)} \circ T_{n(k)}(f)\right|=\left|T_{n(k)} f(p(k))\right|>\varepsilon$. Let $\phi \in C^{*}(N)^{\prime}$ be a weak-* cluster point of the set $\left\{\varepsilon_{p(k)} \circ T_{n(k)}: k=1,2, \ldots\right\}$. By $1.2, \phi \in m_{T}$, and clearly $|\phi(f)| \geqq \varepsilon$; so $f \notin Z_{T}$.

(b) implies (c). Suppose that $\lim _{n \rightarrow \infty}\left(T_{n}\right)_{1} f^{*}=0$. Then $f^{*}=\lim _{n \rightarrow \infty}\left(f^{*}-\left(T_{n}\right)_{1} f^{*}\right)$ uniformly, and it suffices to show that for all $n$,

$$
f^{*}-\left(T_{n}\right)_{1} f^{*} \in \text { linear hull of }\left\{T_{1} g^{*}-g^{*}: g \in C^{*}(N)\right\} \text {. }
$$

But, as in Lemma 3.1 of [4],

$$
f^{*}-\left(T_{n}\right)_{1} f^{*}=f^{*}-(1 / n) \sum_{k=1}^{n} T_{1}^{k} f^{*}=(1 / n) \sum_{k=1}^{n}\left(f^{*}-T_{1}^{k} f^{*}\right),
$$

and each term may be written

where $g_{j}^{*}=T_{1}^{j} f^{*}$.

$$
\begin{aligned}
f^{*}-T_{1}^{k} f^{*} & =\sum_{j=0}^{k-1} T_{1}^{j} f^{*}-T_{1}^{j+1} f^{*} \\
& =\sum_{j=0}^{k-1}\left(g_{j}^{*}-T_{1} g_{j}^{*}\right),
\end{aligned}
$$

(c) implies (a). If $\phi \in m_{T}$, then $\phi(T g-g)=0$ for all $g \in C^{*}(N)$. If (c) holds, then given $\varepsilon>0$, there exists $g \in C^{*}(N)$ with $\left\|f^{*}-\left(T_{1} g^{*}-g^{*}\right)\right\|<\varepsilon$.

Hence there exists $n_{0}$ such that $n \geqq n_{0}$ implies that $|f(n)-(T g-g)(n)|<\varepsilon e(n)$, where $e$ is the unit function; whence, by positivity of each $\phi \in m_{T}$, we have

$$
\begin{aligned}
|\phi(f)| & =|\phi(f)-\phi(T g-g)| \\
& =|\phi((f)-(T g-g))| \\
& \leqq \varepsilon \phi(e)=\varepsilon .
\end{aligned}
$$

Since $\varepsilon>0$ is arbitrary, we have $\phi(f)=0$ for all $\phi \in m_{T}$; so $f \in Z_{T}$. 
2. The conditions (I) and (II).

2.1. THEOREM (cf. Theorems 23 and 24 of [8]). The following are equivalent:

(a) $V_{T} \subset C_{S}$ consistently,

(b) $\left\|S_{1} \circ T_{1}-S_{1}\right\|=0$, i.e. $S_{1} \circ T_{1}=S_{1}$ on $C\left(N^{*}\right)$.

Proof. (b) implies (a). By (b) and (c) of Lemma 1.3, it is enough to show that $Z_{T} \subset K(S)$. If $k=T f-f$ for some $f \in C^{*}(N)$, then (b) implies that $S k \in C_{0}$; whence $k \in K(S)$. By Lemma 1.4, if $g \in V_{T}$ and $\varepsilon>0$, then there exists a function $h$ which is a finite linear combination of functions of the form $T f-f$, and such that $\left\|g^{*}-h^{*}\right\|<\varepsilon$. Hence there exists $n_{0}$ such that $n \geqq n_{0}$ implies that $|g(n)-h(n)|<\varepsilon$. Since $h \in K(S)$,

$$
\varlimsup_{n \rightarrow \infty}|S g(n)| \leqq \varlimsup_{n \rightarrow \infty}|S(g-h)(n)|+\varlimsup_{n \rightarrow \infty}|S h(n)| \leqq \varepsilon .
$$

Since $\varepsilon>0$ is arbitrary, it follows that $g \in K(S)$.

(a) implies (b). If $V_{T} \subset C_{S}$ consistently, then $Z_{T} \subset K(S)$. Hence if $f \in C^{*}(N)$, then $T f-f \in Z_{T} \subset K(S)$; so $(S \circ T-S)(f)=S(T f-f) \in C_{0}$. It follows that the matrices $S \circ T$ and $S$ are equivalent; whence $\left\|S_{1} \circ T_{1}-S_{1}\right\|=0$.

2.2. THEOREM (cf. Theorem 3.3 of [4]). The following are equivalent:

(a) $V_{T} \subset V_{S}$ consistently,

(b) $\lim _{n \rightarrow \infty}\left\|\left(S_{n}\right)_{1} \circ T_{1}-\left(S_{n}\right)_{1}\right\|=0$.

Proof. (b) implies (a). From 1.3(b) it follows that $V_{T} \subset V_{S}$ consistently iff $Z_{T} \subset Z_{S}$. Now condition (b) implies that $\lim _{n \rightarrow \infty}\left(\left(S_{n}\right)_{1} \circ T_{1}-\left(S_{n}\right)_{1}\right)\left(f^{*}\right)=0$ for each $f \in C^{*}(N)$. If $g \in V_{T}$ and $g=T h-h$ for some $h$, then we have immediately that $\lim _{n \rightarrow \infty}\left\|\left(S_{n}\right)_{1} g^{*}\right\|=0$; so $g \in V_{S}$, by 1.4. In general, if $f \in V_{T}$ and $\varepsilon>0$, then by 1.4 there is a $g \in V_{T}$ which is a finite linear combination of elements of the form $T h-h$, and such that $\left\|f^{*}-g^{*}\right\|<\varepsilon$. Clearly, $\lim _{n \rightarrow \infty}\left\|\left(S_{n}\right)_{1} g^{*}\right\|=0$, and so there exists $n_{0}$ such that $n \geqq n_{0}$ implies (since $\left\|\left(S_{n}\right)_{1}\right\|=1$ for all $n$ ) that

$$
\left\|\left(S_{n}\right)_{1} f^{*}\right\| \leqq\left\|\left(S_{n}\right)_{1}\left(f^{*}-g^{*}\right)\right\|+\left\|\left(S_{n}\right)_{1} g^{*}\right\|<2 \varepsilon .
$$

Hence $\lim _{n \rightarrow \infty}\left\|\left(S_{n}\right)_{1} f^{*}\right\|=0$; so $f \in V_{S}$, by 1.4 .

(a) implies (b). If $V_{T} \subset V_{S}$ consistently, then $Z_{T} \subset Z_{S}$, or, equivalently, $m_{S} \subset m_{T}$. We show that this implies that

$$
\lim _{n \rightarrow \infty}\left\|\left(\left(S_{n}\right)_{1} \circ T_{1}-\left(S_{n}\right)_{1}\right) f^{*}\right\|=0
$$

for each $f \in C^{*}(N)$. The desired conclusion then follows from Lemma 2.3 below.

We suppose, to obtain a contradiction, that there exist $f \in C^{*}(N), \varepsilon>0$, and $n(1)<n(2)<\ldots$ such that

$$
\left\|\left(\left(S_{n(k)}\right)_{1} \circ T_{1}-\left(S_{n(k)}\right)_{1}\right) f^{*}\right\|>\varepsilon \quad(k=1,2, \ldots) .
$$


Then there exist $p(1)<p(2)<\ldots$, with $\left|S_{n(k)}(T f-f)(p(k))\right|>\varepsilon, k=1,2, \ldots$ By Lemma 1.2 , if $\phi$ is a weak-* cluster point in $C^{*}(N)^{\prime}$ of the functionals $\left\{\varepsilon_{p(k)} \circ S_{n(k)}: k=1,2, \ldots\right\}$, then $\phi \in m_{S}$, and clearly $|\phi(T f-f)| \geqq \varepsilon$. This implies that $\phi \notin m_{T}$, contradicting our assumption that $m_{S} \subset m_{T}$.

2.3. Lemma. Let $A_{1}, A_{2}, \ldots$ be operators on $C\left(N^{*}\right)$ induced by matrix operators. Then $\lim _{n \rightarrow \infty}\left\|A_{n}\right\|=0$ iff $\lim _{n \rightarrow \infty}\left\|A_{n} f\right\|=0$ for each $f \in C\left(N^{*}\right)$.

Proof. Sufficiency is obvious. For necessity, suppose that $\left\|A_{n}\right\| \rightarrow 0$ as $n \rightarrow \infty$. Taking subsequences if need be, we may assume that, for some $\varepsilon>0,\left\|A_{n}\right\| \geqq \varepsilon$ for all $n$. Let $T^{n}$ be a matrix operator inducing $A_{n}$, which we assume to be in truncated form, and let $T_{m}^{n}$ be the $m$ th row of $T^{n}$, considered as a linear functional (with finite support, because of truncation) on $C^{*}(N)$.

Now for each $n$ there exists $f_{n} \in C\left(N^{*}\right)$ with $\left\|f_{n}\right\| \leqq 1$ and $\sup \left\{\left|A_{n} f_{n}(w)\right|: w \in N^{*}\right\}>\varepsilon$. Let $g_{n}$ be an extension of $f_{n}$ to all $\beta N$ such that $\left\|g_{n}\right\| \leqq 1$. Now choose $n(1)$ such that $\left|T_{n(1)}^{1} g_{1}\right|>\varepsilon$, with, say, support $\left(T_{n(1)}^{1}\right) \subset[0, N(1))$; choose $n(2)$ with $\left|T_{n(2)}^{2} g_{2}\right|>\varepsilon$, and support $\left(T_{n(2)}^{2}\right) \subset[N(1), N(2))$; choose $n(3)$ with $\left|T_{n(3)}^{1} g_{1}\right|>\varepsilon$, and $\operatorname{support}\left(T_{n(3)}^{1}\right) \subset[N(2), N(3))$; choose $n(4)$ with $\left|T_{n(4)}^{2} g_{2}\right|>\varepsilon$, and support $\left(T_{n(4)}^{2}\right) \subset[N(3), N(4))$; choose $n(5)$ with $\left|T_{n(5)}^{3} g_{3}\right|>\varepsilon$, and support $\left(T_{n(5)}^{3}\right) \subset[N(4), N(5)) ;$ and so on in the pattern 1-2, 1-2-3, $1-2-3-4$, etc.

Define $g \in C^{*}(N)$ so that $g$ agrees with $g_{1}$ on $[0, N(1))$, with $g_{2}$ on [N(1), $N(2)$ ), with $g_{1}$ on [N(2), $N(3)$ ), with $g_{2}$ on [N(3), $N(4)$ ), with $g_{3}$ on [N(4), $N(5)$ ), and so on. Then clearly $\limsup _{m \rightarrow \infty}\left|T_{m}^{n} g\right| \geqq \varepsilon$ for each $n$; hence, if $f$ denotes the extension of $g$ to $\beta N \mid N$, then $\left\|A_{n} f\right\| \geqq \varepsilon$ for each $n$, so that $\lim _{n \rightarrow \infty}\left\|A_{n} f\right\|=0$ fails. This completes the proof.

\section{Sufficiency for (III).}

3.1. Notation. As a linear function, $\varepsilon_{n} \circ T$ is the same as the $n$th row of $T$. If $T=\left(t_{m n}\right)$ and $S=\left(s_{m n}\right)$ are non-negative regular (and assumed to be in truncated form), we write

$$
d\left(\varepsilon_{m} \circ S, \varepsilon_{n} \circ T\right)=\sum_{k}\left|s_{m k}-t_{n k}\right| \text {. }
$$

We write $S_{n, p}=(1 / n) \sum_{k=1}^{n} \varepsilon_{p} \circ S^{k}$, where $S^{k}$ is the $k$ th iterate of $S$. If $L \subset C^{*}(N)^{\prime}$, then $L_{c}$ is the weak-* closed convex hull of $L$ in $C^{*}(N)^{\prime}$.

Remark. In this section we deal with the inclusion $C_{T} \subset V_{S}$ consistently. The following lemma gives some convenient restatements of this inclusion.

\subsection{LEMMA.}

(a) $C_{T} \subset V_{S}$ consistently iff $K(T) \subset Z_{S}$.

(b) $K(T) \subset Z_{S}$ iff $K(T)^{*} \subset\left(Z_{S}\right)^{*}$ (where $K(T)^{*}=\left\{f^{*}: f \in K(T)\right\}$, etc.),

(c) $K(T)^{*} \subset\left(Z_{S}\right)^{*}$ iff $m_{S}$ is contained in the closed linear hull in $C^{*}(N)^{\prime}$ of $\left\{\varepsilon_{p} \circ T_{1}: p \in N^{*}\right\}$. 
Proof. (a) Immediate from (b) and (c) of 1.3. (b) Immediate from the definitions.

(c) If $\phi \in m_{T}$, and $\phi^{\prime}$ is the Borel measure on $\beta N$ representing $\phi$, then since $\phi(f)=0$ for all $f \in c_{0}, \phi^{\prime}$ is supported by $N^{*}$. Since $K(T)^{*}=\left\{f^{*}: T_{1} f^{*}=0\right\}$, where $T_{1}$ is the operator on $C\left(N^{*}\right)$ induced by $T$, we have $f^{*} \in K(T)^{*}$ iff $\varepsilon_{p} \circ T_{1}\left(f^{*}\right)=0$ for all $p \in N^{*}$; and $f^{*} \in\left(Z_{S}\right)^{*}$ iff $\phi^{\prime}\left(f^{*}\right)=0$ for all $\phi \in m_{T}$. By [3, p. 20, (8)] the following are equivalent: (i) $\varepsilon_{p} \circ T_{1}\left(f^{*}\right)=0$ for all $p \in N^{*}$ implies that $\phi^{\prime}\left(f^{*}\right)=0$ for all $\phi \in m_{T}$; (ii) $\left\{\phi^{\prime}: \phi \in m_{S}\right\}$ is contained in the weak-* closed linear hull of $\left\{\varepsilon_{p} \circ T_{1}: p \in N^{*}\right\}$. This completes the proof of the lemma.

It is part (c) of the lemma that suggests the form of Theorems 3.4 and 4.3 below. Our problem is to translate (c) into a condition involving the rows of the matrices $S$ and $T$.

3.3. Lemma.

$$
\left\{\varepsilon_{p} \circ T_{1}: p \in N^{*}\right\}_{c}=\bigcap_{n=1}^{\infty} W_{n},
$$

where $W_{n}=\left\{\varepsilon_{m} \circ T: m=n, n+1, \ldots\right\}_{c}$.

Proof. According to Theorem 2 in Jerison's paper [6], if $K_{n}$ is a sequence of compact convex sets in a locally convex space $E$, with $K_{n+1} \subset K_{n}$, and $A_{n}$ is the set of extreme points of $K_{n}$, then

$$
\bigcap_{n} K_{n}=\text { closed convex hull of } \bigcap_{n} A_{n} \text {. }
$$

Let $B_{n}=\left\{\varepsilon_{m} \circ T: m \geqq n\right\} \cup\left\{\varepsilon_{p} \circ T_{1}: p \in N^{*}\right\}$. It follows easily from a theorem of Milman (Theorem 1 of [6]) that $B_{n}$ is the set of extreme points of $W_{n}$. (Milman's theorem says that, if $C$ is compact and convex and $S \subset C$, then the closure of $S$ contains all the extreme points of $C$ iff $\sup \{f(x): x \in C\}=\sup \{f(x): x \in S\}$ for each continuous linear functional $f$.) Jerison's theorem yields $\bigcap_{n} W_{n}=\left(\bigcap_{n} B_{n}\right)_{c}$. But it is easy to see that $\bigcap_{n} B_{n}=\left\{\varepsilon_{p} \circ T_{1}: p \in N^{*}\right\}$.

Definition. $R_{m}$ is the norm closed convex hull of $\left\{\varepsilon_{n} \circ T: n=m, m+1, \ldots\right\}$ (cf. Remark 4.2).

3.4. THEOREM. If for each $m, \lim _{n, p \rightarrow \infty} d\left(S_{n, p}, R_{m}\right)=0$, then $C_{T} \subset V_{S}$ consistently.

Proof. Since $R_{m} \subset W_{m}$, the condition of the theorem implies that $\lim _{n, p \rightarrow \infty} d\left(S_{n, p}, W_{m}\right)=0$ for each $m$. We shall show that this implies that $C_{T} \subset V_{S}$.

As noted in 3.2, we must show that $m_{s} \subset\left\{\varepsilon_{p} \circ T: p N^{*}\right\}_{c}$ or, by Lemma 3.3, that $m_{s} \subset \bigcap_{m} W_{m}$. Since $m_{s}$ is the weak-* closed convex hull of functionals of the form

$$
\phi=\lim \left\{\varepsilon_{p(a)} \circ S_{n(a)}: a \in A\right\}=\lim \left\{S_{n(a), p(a)}: a \in A\right\},
$$

with $\lim \{p(a): a \in A\}=\lim \{n(a): a \in A\}=\infty$, it suffices to show that each such $\phi$ belongs to $\bigcap_{m} W_{m}$. By hypothesis, and since $n(a) \rightarrow \infty, p(a) \rightarrow \infty$, we have $\lim \left(d\left(S_{n(a), p(a)}, W_{m}\right): a \in A\right)=0$ for each $m$. Fix $m$ and choose $\phi_{a} \in W_{m}$ such that $\lim \left\{\left\|S_{n(a), p(a)}-\phi_{a}\right\|: a \in A\right\}=0$. Since $W_{m}$ 
is weak-* compact, there exist $\phi_{1} \in W_{m}$ and a subnet $\left\{\phi_{b}: b \in B\right\}$ such that $\phi_{1}=\lim \left\{\phi_{b}: b \in B\right\}$. Now if $f \in C^{*}(N)$, then

$$
\begin{aligned}
\left|\phi(f)-\phi_{1}(f)\right| & =\lim \left\{\left|S_{n(b), p(b)} f-\phi_{b} f\right|: b \in B\right\} \\
& \leqq \lim \left\{\left\|S_{n(b), p(b)}-\phi_{b}\right\|: b \in B\right\}\|f\|=0 .
\end{aligned}
$$

Hence $\phi=\phi_{1} \in W_{m}$ for arbitrary $m$.

4. Necessity for (III). In this section we prove, under a restriction on $T$, a converse to 3.4. The author does not know to what degree the restriction can be weakened. First we need a technical lemma.

4.1. LeMMA. For each $m, \lim _{n, p \rightarrow \infty} d\left(S_{n, p}, R_{m}\right)=0$ iff $\lim _{n, p \rightarrow \infty} d\left(S_{n, p}, W_{m}\right)=0$.

Proof. Sufficiency has already been noted. For necessity, assume that $\lim d\left(S_{n, p}, W_{m}\right)=0$. We show that for fixed $m, n$ and $p$, if $d\left(S_{n, p}, W_{m}\right)<\alpha<\frac{1}{2}$, then $d\left(S_{n, p}, R_{m}^{n, p \rightarrow \infty}<<4 \alpha\right.$. The desired result follows easily from this.

Write $\psi=S_{n, p}$. Since our matrices are assumed truncated,

$$
\text { support }(\psi) \subset[0, N] \text { for some } N \text {. }
$$

Now there is a $\phi \in W_{m}$ with $d(\phi, \psi)<\alpha$. For some net, $\phi=$ weak-* $\lim (a \in A) \phi_{a}$, where $\phi_{a}=\sum_{i} t_{a, i} T_{p(a, i)}$, and the combination is convex. Write

$$
\phi_{a}=\Sigma^{\prime} t_{a, i} T_{p(a, i)}+\Sigma^{\prime \prime} t_{a, i} T_{p(a, i)}=\phi_{1, a}+\phi_{2, a}
$$

where $\Sigma^{\prime \prime}$ is summation over those terms such that the support of $T_{p(a, i)}$ is disjoint from $[0, N]$, and $\Sigma^{\prime}$ is summation over the rest of the terms. Let $g$ be the characteristic function of $[N+1, \infty)$. Then $\psi(g)=0$, so that

$$
|\phi(g)|=|\phi(g)-\psi(g)| \leqq\|\phi-\psi\|\|g\|<\alpha .
$$

Hence, for some $a_{0} \in A, a \geqq a_{0}$ implies (since $T \geqq 0$ ) that

$$
k(a)=\Sigma^{\prime \prime} t_{a, i}=\left|\phi_{2, a}(g)\right| \leqq\left|\phi_{a}(g)\right|<\alpha .
$$

Let $\eta_{a}=\Sigma^{\prime} t_{a, i}(1-k(a))^{-1} T_{p(a, i)}$, a convex combination of rows of $T$, each of whose supports meet $[0, N]$. Now it follows from the truncated form of $T$ that, since the support of each $T_{p(a, i)}$ involved in the sum meets $[0, N]$, there exists fixed $M \geqq N$ such that

Hence

$$
\text { support } T_{p(a, l)} \subset[0, M] \text {. }
$$

$$
\operatorname{support}\left(\eta_{a}\right) \subset[0, M] \text { for } a \geqq a_{0} .
$$
Then

Let $\eta$ be a weak-* cluster point of the $\eta_{a}$, and $\eta_{b}$ a subnet of $\eta_{a}$ which converges to $\eta$.

$$
\text { support }(\eta) \subset[0, M] \text {. }
$$


For each $b$,

$$
\begin{aligned}
\left\|\eta_{b}-\phi_{b}\right\| & =\left\|(1-k(b))^{-1} \phi_{1, b}-\phi_{1, b}-\phi_{2, b}\right\| \\
& \leqq\left((1-k(b))^{-1}-1\right)\left\|\phi_{1, b}\right\|+\left\|\phi_{2, b}\right\| \\
& <k(b)(1-k(b))^{-1}+k(b) \\
& <3 k(b)<3 \alpha,
\end{aligned}
$$

since $\alpha<\frac{1}{2}$. Hence $\|\eta-\phi\| \leqq 3 \alpha$, and $\|\eta-\psi\|<4 \alpha$. But

support $\eta \subset[0, M]$ and support $\eta_{b} \subset[0, M]$.

This implies that the convergence of $\eta_{b}$ to $\eta$ is essentially finite-dimensional. But in this case weak-* convergence is equivalent to norm convergence [3, p. 39]; so $\eta \in R_{m}$, and $d\left(\psi, R_{m}\right) \leqq$ $d(\psi, \eta)<4 \alpha$, where $\psi=S_{n, p}$.

4.2. Remark. It should be noted that each element of $R_{m}$ is a functional on $C(\beta N)$ whose support is contained in $N$, hence may be represented by a vector in $l^{1}$. This is by no means the case for $W_{m}$, and is the reason why $R_{m}$ is preferable to $W_{m}$.

4.3. THeOREM. Assume that the induced operator $T_{1}$ maps $\left\{f \in C\left(N^{*}\right): f \geqq 0\right\}$ onto itself. If $C_{T} \subset V_{S}$ consistently, then $\lim _{n, p \rightarrow \infty} d\left(S_{n, p}, R_{m}\right)=0$ for all $m$.

Proof. First we show that, under our hypotheses on $T$, if $\phi \in C\left(N^{*}\right)^{\prime}$ (the dual space of $\left.C\left(N^{*}\right)\right), \phi(e)=1, \phi \geqq 0$ and $\phi \in$ weak-* $^{*}$ closed linear hull of $\left\{\varepsilon_{w} \circ T_{1}: w \in N^{*}\right\}$, then $\phi \in\left\{\varepsilon_{w} \circ T_{1}: w \in N^{*}\right\}_{c}$. But our hypotheses imply readily that the range of $T_{1}$ is all of $C\left(N^{*}\right)$; hence the range is closed, and it follows from [5, Theorem 2, p. 487] that the range of the adjoint map $T_{1}^{\prime}$ consists of those $\psi \in C\left(N^{*}\right)^{\prime}$ for which $T_{1} f=0$ implies that $\psi(f)=0$. Since our $\phi$ satisfies this latter condition, we have $\phi=\psi \circ T_{1}$ for some $\psi \in C\left(N^{*}\right)^{\prime}$. Now since $T_{1}(e)=e$, we have $\psi(e)=\psi\left(T_{1} e\right)=\phi(e)=1$. If $f \geqq 0$, then $f=T_{1} g$ for some $g \geqq 0$, so that $\psi(f)=\psi\left(T_{1} g\right)=\phi(g) \geqq 0$. It follows that $\psi$ is the weak-* limit of functionals of the form $\sum t_{i} \varepsilon_{w(i)}$, where $w(I) \in N^{*}$ and the combination is convex. Since the adjoint $T_{1}^{\prime}$ is weak-* continuous, it follows that $\phi$ is the weak-* limit of functionals of the form $\sum t_{i} \varepsilon_{w(i)} \circ T_{1}$, so that $\phi \in\left\{\varepsilon_{w} \circ T_{1}: w \in N^{*}\right\}_{c}$.

Suppose now that for some $m, d\left(S_{n, p}, R_{m}\right)$ does not go to 0 . Then, by Lemma 4.1, neither does $d\left(S_{n, p}, W_{m}\right)$, so there exist $\varepsilon>0, n(1)<n(2)<\ldots$ and $p(1)<p(2)<\ldots$ with $d\left(S_{n(k), p(k)}, W_{m}\right)>\varepsilon$ for all $k$. Let $\phi$ be a weak-* cluster point of the functionals $\left\{S_{n(k), p(k)}\right\}$. Then $\phi \in m_{S}$ by Lemma 1.2. Clearly, $d\left(\phi, W_{m}\right) \geqq \varepsilon$, and since, by Lemma 3.3, $\left\{\varepsilon_{w} \circ T: w \in N^{*}\right\}_{c} \subset W_{m}$, we have $\phi \notin\left\{\varepsilon_{w} \circ T: w \in N^{*}\right\}_{c}$. From the first part of the proof it follows that $\phi$ does not belong to the weak-* closed linear hull of $\left\{\varepsilon_{w} \circ T: w \in N^{*}\right\}$; so by Lemma 3.2 the inclusion $C_{T} \subset V_{S}$ consistently fails. This completes the proof.

\section{Examples.}

5.1. For an example in which inclusion (I) fails but inclusion (II) holds, let $S$ be the shift matrix and let $T=S^{2}$. Clearly, $V_{T} \subset V_{S}$ consistently, while the criterion of Theorem 2.1 shows that $V_{T} \subset C_{S}$ consistently fails. 
5.2. For an example in which (II) fails while (III) holds, let $S$ and $R$ be any pair of matrices such that $C_{R} \subset V_{S}$ consistently. From $R$ we form a new matrix $T$ by letting the odd rows of $T$ be all the rows of $R$, and choosing the even rows so as to cause the inclusion $V_{T} \subset V_{S}$ to fail. Then $C_{r} \subset C_{R} \subset V_{S}$, so that (III) continues to hold.

5.3. The extra hypothesis on $T$ in Theorem 4.3 is actually fulfilled by a reasonably large class of matrices. For instance, let $T$ be a non-negative regular matrix whose rows have disjoint support, i.e. if $m \neq q$, then $t_{m k} \neq 0$ implies that $t_{q k}=0$. Let the support of the $m$ th row be contained in the interval $[k(m), k(m+1)$ ), where, for distinct $m$, the intervals are disjoint. If $\left\{r_{n}\right\}$ is any bounded sequence of non-negative constants, define $f \in C^{*}(N)$ by the formula $f(k)=r_{m}$ whenever $k \in[k(m), k(m+1)$ ), and let $f$ be 0 elsewhere. Then, assuming each row sum of $T$ is 1 , we have $T f(m)=r_{m}$ for all $m$. Thus $T$ maps the space of non-negative elements of $C^{*}(N)$ onto itself, and it is easy to see that $T_{1}$ does the same for $C\left(N^{*}\right)$.

\section{REFERENCES}

1. R. Atalla, On the multiplicative behavior of regular matrices; to appear.

2. R. Atalla and J. Bustoz, Sequential cores and a theorem of R. R. Phelps, Proc. Amer. Math. Soc. 21 (1969), 36-42.

3. M. M. Day, Normed linear spaces (New York, 1962).

4. D. Dean and R. A. Raimi, Permutations with comparable sets of invariant means, Duke Math. J. 27 (1960), 467-480.

5. N. Dunford and J. Schwartz, Linear operators, Vol. I (New York, 1958).

6. M. Jerison, A property of extreme points of compact convex sets, Proc. Amer. Math. Soc. 5 (1954), 782-783.

7. G. M. Petersen, Regular matrix transformations (McGraw-Hill, London, 1966).

8. R. A. Raimi, Invariant means and invariant matrix methods of summability, Duke Math.J. 30 (1963), 81-94.

\section{OHIO UNIVERSITY}

ATHENS, OHIO 